\title{
Hilar Malignant Biliary Obstruction Treated with Four Metallic Stents Involving a New Slim Device
}

\author{
Yuki Noguchi ${ }^{1}$, Mitsuru Sugimoto ${ }^{1}$, Yuichiro Kiko ${ }^{2}$, Tadayuki Takagi ${ }^{1}$, Rei Suzuki ${ }^{1}$, \\ Naoki Konno ${ }^{1}$, Hiroyuki Asama ${ }^{1}$, Yuki Sato ${ }^{1}$, Hiroki Irie ${ }^{1}$, Jun Nakamura ${ }^{1,3}$, Mika Takasumi ${ }^{1}$, \\ Minami Hashimoto ${ }^{1,3}$, Tsunetaka Kato ${ }^{1,3}$, Ryoichiro Kobashi ${ }^{1}$, Yuko Hashimoto ${ }^{2}$, \\ Takuto Hikichi ${ }^{3}$ and Hiromasa Ohira ${ }^{1}$
}

\begin{abstract}
:
Endoscopic hilar multiple stenting is challenging. A 68-year-old patient had self-expandable metallic stents (SEMSs) inserted for unresectable hilar malignant biliary obstruction. After the SEMSs were inserted into the left hepatic duct and bile duct branch of segment (B) 6, a new SEMS with a wide mesh and slim delivery system was inserted into the right anterior hepatic duct. However, liver abscess and dilated B7 were observed on computed tomography; therefore, an additional new SEMS was quickly and easily inserted into B7. After the placement of these four SEMSs, the liver abscess improved. The new SEMS was effective for hilar multiple biliary drainage.
\end{abstract}

Key words: hilar malignant biliary obstruction, bilateral biliary drainage, self-expandable metallic stent

(Intern Med 60: 1871-1876, 2021)

(DOI: 10.2169/internalmedicine.6356-20)

\section{Introduction}

For biliary ductal cancer, biliary drainage is needed in order to administer chemotherapy. While preoperative biliary drainage is controversial, it is recommended for patients with hilar biliary obstruction who need extensive hepatectomy (1-3). The methods of biliary drainage include percutaneous transhepatic biliary drainage (PTBD) and endoscopic biliary drainage. However, PTBD carries risks of damaging the hepatic vessels and causing peritoneal seeding $(4,5)$. Therefore, endoscopic biliary drainage has become the first choice.

For unresectable biliary cancer, uncovered self-expandable metallic stents (SEMSs) are recommended for biliary drainage. The patency period, successful drainage, and overall survival are better with uncovered SEMSs than with plastic stents (6-10). Furthermore, whether unilateral drainage or bilateral drainage should be performed for hilar biliary cancer is controversial (11-19). However, there are some patients who need bilateral biliary drainage.

We experienced a case in which four uncovered SEMSs were inserted to treat liver abscess. In a retrospective study, six patients with hilar biliary obstruction in whom four uncovered SEMSs were placed were reported (20). However, the details concerning the insertion of the four uncovered SEMSs were not described. In addition, multistenting using uncovered SEMSs is sometimes difficult. We herein report how we easily achieved endoscopic multistenting with four uncovered SEMSs using a new device.

\section{Case Report}

A 68-year-old man visited the previous hospital with yellowing of the skin. Elevated serum hepatobiliary enzymes were observed on a blood test. He was referred to our hospital for an intensive examination and treatment. He had a history of hypertension. He had no fever, and his abdomen was soft and flat. He felt mild tenderness in the right hypochondriac region.

${ }^{1}$ Department of Gastroenterology, School of Medicine, Fukushima Medical University, Japan, ${ }^{2}$ Department of Diagnostic Pathology, School of Medicine, Fukushima Medical University, Japan and ${ }^{3}$ Department of Endoscopy, Fukushima Medical University Hospital, Japan Received: September 20, 2020; Accepted: December 7, 2020; Advance Publication by J-STAGE: February 1, 2021 Correspondence to Dr. Mitsuru Sugimoto, kita335@fmu.ac.jp 

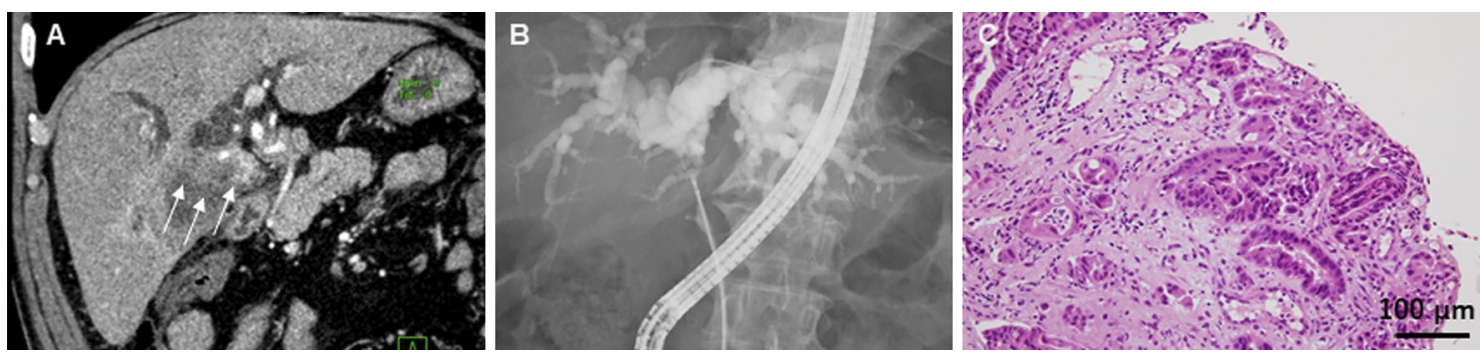

Figure 1. Image findings and the diagnosis. A: A gallbladder tumor with common bile duct invasion was observed in contrast-enhanced CT (arrows). B: Common bile duct stricture was seen during ERC. A biliary ductal biopsy was performed. C: The biliary biopsy specimen was diagnosed as adenocarcinoma. Fibrosis was observed in the overall specimen. The tumor cells had irregular tubular structures and invasive proliferation. CT: computed tomography, ERC: endoscopic retrograde cholangiography

On laboratory examinations, elevated serum hepatobiliary enzymes were observed (aspartate transaminase $68 \mathrm{U} / \mathrm{L}$, alanine transaminase $171 \mathrm{U} / \mathrm{L}$, alkaline phosphatase 1,038 $\mathrm{U} / \mathrm{L}, \gamma$-glutamyltransferase, $388 \mathrm{U} / \mathrm{L}$, total bilirubin $11.4 \mathrm{mg} /$ $\mathrm{dL}$, and direct bilirubin $8.8 \mathrm{mg} / \mathrm{dL}$ ). In addition, serum carbohydrate antigen 19-9 was elevated to $127.1 \mathrm{U} / \mathrm{mL}$.

On contrast-enhanced CT (CE-CT), a heterogeneously enhanced tumor was observed in the gallbladder, and the tumor invaded the common bile duct (Fig. 1A). The intrahepatic biliary duct was dilated by the tumor. Endoscopic retrograde cholangiography (ERC) showed stricture of the common bile duct, and a biliary ductal biopsy was performed (Fig. 1B). The biliary ductal biopsy specimen was diagnosed as adenocarcinoma (Fig. 1C). After the jaundice was improved by endoscopic biliary drainage, the patient underwent surgery. Small liver metastases were observed; therefore, tumor resection was not performed. The final diagnosis of this lesion was gallbladder cancer, c stage IV (cT 3 N1M1) according to the Union for International Cancer Control classification, 8th edition (21).

Initially, a 6-Fr endoscopic nasobiliary drainage (ENBD) tube (Flexima ${ }^{\mathrm{TM}}$ ENBD catheter; Boston Scientific Japan, Tokyo, Japan) was inserted into the left hepatic duct, and a 7-Fr 12-cm plastic stent (Flexima ${ }^{\mathrm{TM}}$ Plus; Boston Scientific Japan) was inserted into the right posterior hepatic duct. Ten days after the plastic stent and ENBD tube had been inserted, he developed a fever. On abdominal computed tomography (CT), dilation of the right anterior hepatic duct was observed. Therefore, a 5-Fr ENBD tube (Flexima ${ }^{\mathrm{TM}}$ ENBD catheter; Boston Scientific Japan) was added to the right anterior hepatic duct. However, liver metastases were observed during surgery, as described above. Therefore, the drainage tube was exchanged for an uncovered SEMS. During ERC, the hilar biliary duct was divided into four hepatic ducts (left, right anterior, bile duct branch of segment (B) 6, and B7) (Fig. 2A). As three drainage catheters had been inserted before surgery, we tried to achieve drainage of more than three divided haptic ducts. All SEMSs were placed in stent-in-stent form. First, a Niti-S large cell uncovered metallic stent D-type $(10 \mathrm{~mm} \times 10 \mathrm{~cm}$; Taewoong Medical,
Gyeoenggi-do, Korea) was inserted into the left hepatic duct. Second, the same stent $(10 \mathrm{~mm} \times 8 \mathrm{~cm})$ was inserted into B6. Third, the Niti-S large cell slim delivery (Taewoong Medical) was inserted into the right anterior hepatic duct (Fig. 2B) after dilation of the selected cell using a balloon catheter. The balloon catheter used in this report was a REN $6 \mathrm{~mm}$ (KANEKA MEDICAL PRODUCTS, Osaka, Japan).

However, one day after the three uncovered biliary SEMSs had been inserted, the patient showed a fever. He was administered antibiotic drugs. Approximately one week after SEMS insertion, a liver abscess with dilated B7 was observed by CT (Fig. 2C). Therefore, one more ERC was performed. The guidewire and catheter proceeded to B7, and infected bile juice was suctioned. After B7 was enhanced (Fig. 2D), the Niti-S large cell slim delivery (Taewoong Medical) was inserted into B7 (Fig. 2E). In our hospital, 8Fr Niti-S large cell uncovered metallic stents (Taewoong Medical) were used for as many hilar malignant biliary obstructions as possible because the stent consists of thick wire, and the stent has been confirmed to have a sufficient radial force in previous reports (22-24). As the radial force of the previously inserted stent is strong, the additional SEMS insertion was easy. If a guidewire or an endoscopic retrograde cholangiopancreatography (ERCP) catheter proved difficult to pass through the mesh of the previously inserted SEMS, a 6 Fr Niti-S slim type (Taewoong Medical) was selected. The order of SEMS insertion was as follows: left hepatic duct first, right posterior hepatic duct second, and right anterior hepatic duct third. However, the right posterior hepatic ducts (B6 and B7) were divided by the tumor in this case. Therefore, in the right anterior hepatic duct, the SEMS was inserted earlier than the B7 to maintain the drainage of the larger liver volume.

Forty-three days after four SEMSs were inserted, the liver abscess was improved (Fig. 2F). Chemotherapy (gemcitabine and cisplatin) was initiated, and the recurrence of the liver abscess was not observed. Antibiotic drugs were administered for 77 days [intravenous tazobactam/piperacillin (TAZ/ PIPC) $13.5 \mathrm{~g} /$ day for 5 days, intravenous and oral levofloxacin (LVFX) $500 \mathrm{mg} /$ day for 72 days] until the liver abscess 

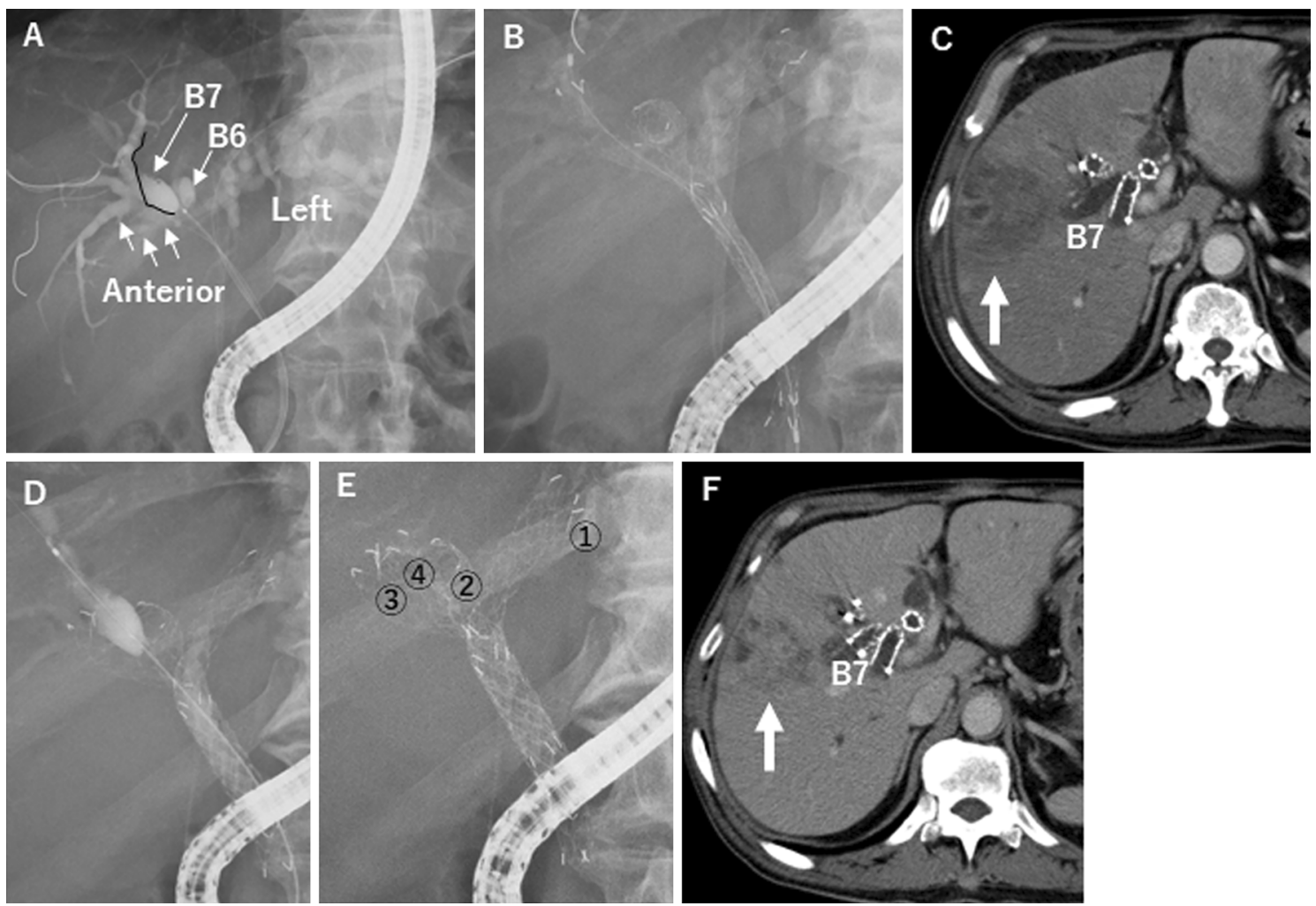

Figure 2. The state of biliary stricture and treatment. A: The hepatic ducts were divided into four branches (left, B6, B7, right anterior) by cancer. The black line represents the border between $\mathrm{B} 7$ and the right anterior hepatic duct. B: The Niti-S large cell stent $D$ type $10 \mathrm{~mm} \times 10 \mathrm{~cm}$ (Taewoong Medical) was inserted in the left hepatic duct. The same stent $(10 \mathrm{~mm} \times 8 \mathrm{~cm})$ was inserted in B6. The new Niti-cell large cell stent with a slim delivery system (Taewoong Medical) was inserted into the right anterior hepatic duct. C: Approximately one week after the three SEMSs had been inserted, dilated B7 and a liver abscess (arrow) were observed on CT. D: ERC was performed, and the guidewire and ERC catheter were inserted in B7. E: Finally, four SEMSs were placed. F: Forty-three days after the four SEMSs had been inserted, improvement of the liver abscess was seen (arrow). All SEMSs were placed in a stent-in-stent fashion. B: The bile duct branch of the segment, SEMS: self-expandable metallic stent, CT: computed tomography, ERC: endoscopic retrograde cholangiography

was not clearly detected by CE-CT.

\section{Discussion}

As mentioned in the introduction, whether or not bilateral biliary drainage should be performed has been the subject of discussion $(11,12,14-17,25)$. The results of two recent randomized controlled trials were contradictory concerning whether unilateral or bilateral biliary drainage is optimal $(18,19)$. In addition to discussions concerning bilateral or unilateral biliary drainage, the liver volume for which biliary drainage is performed has also been reported to be important. Vienne et al. (26) reported that a drained liver volume exceeding $50 \%$ is needed for effective biliary drainage. Takahashi et al. (27) reported that a drained liver volume exceeding $33 \%$ and $50 \%$ was effective in patients with a preserved liver function and impaired liver function, respectively. In the present case, a drained liver volume exceeding 50\% was achieved at the first endoscopic SEMS insertion. However, the dilation of B7 and liver abscess oc- curred, possible because B7 was enhanced by ERC without drainage (11). While B7 was unintentionally enhanced and ultimately drained, a biliary duct in which drainage is not scheduled should not be enhanced.

For biliary drainage with liver abscess, ENBD has been recommended in previous reports (28-32). However, the addition of an SEMS was performed in the present case because the patient had had ENBD catheters in place for a long time. In addition, tumor progression was observed when the SEMSs were placed. Therefore, the liver abscess was treated by an SEMS in order to allow the patient to begin eating and start chemotherapy as soon as possible. The liver abscess improved in response to an additional SEMS placement. However, even if the liver abscess had not been improved by SEMSs, ENBD placement would have been possible. Because an SEMS was finally inserted for the liver abscess, the lumen of the fourth stent was not closed by the other SEMSs.

Previous reports have proposed predictive risk factors for the failure of stent-in-stent SEMS placement $(33,34)$. In 


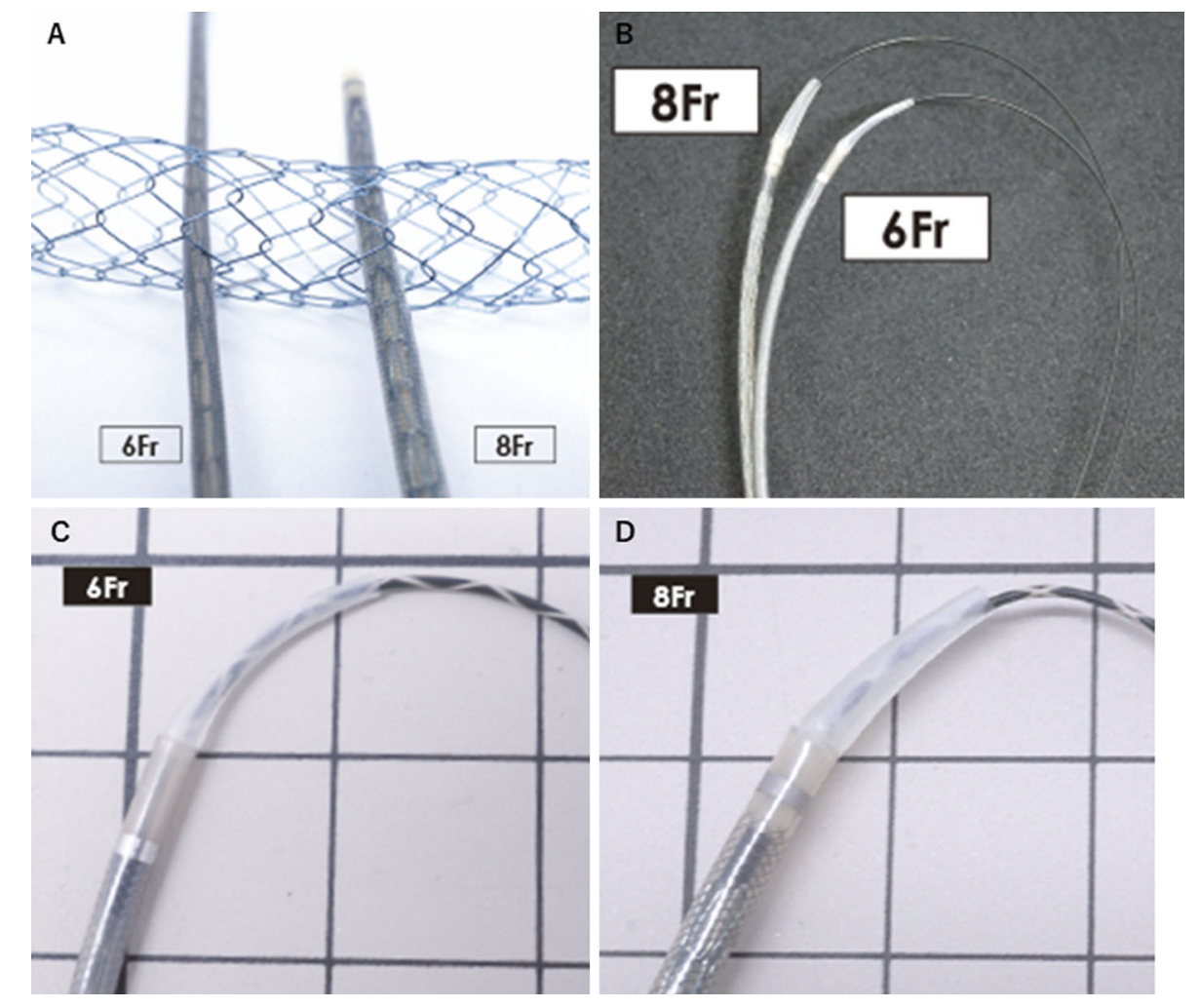

Figure 3. The new SEMS with a slim delivery system. The figures were supplied by Century Medical (Tokyo, Japan). A: The Niti-S large cell stent slim delivery (Taewoong Medical) with a 6-Fr delivery system. The delivery system of the conventional Niti-S large cell D type stent (Taewoong Medical) was 8 Fr. B: The 6-Fr delivery system has good trackability to the guidewire. C, D: At the tip of the 6-Fr delivery system, the step between the delivery system and guidewire becomes lower than that of the delivery system of the conventional 8-Fr delivery system. SEMS: self-expandable metallic stent

those reports, SEMSs with a large mesh and thin delivery were reported to be desirable. The Niti-S large cell slim delivery (Taewoong Medical) has a wide mesh and thin delivery system, which fits the favorable stent condition for the insertion of multiple SEMSs. The Niti-S large cell slim delivery (Taewoong Medical) was used for the third and fourth stents in this study, as shown in Fig. 3 (offered by Century Medical, Tokyo, Japan). The delivery system of the conventional Niti-S large cell stent was 8 Fr; in contrast, the delivery system of the new Niti-S large cell stent slim delivery was 6 Fr (Fig. 3A). The delivery system of the new stent has good trackability to the guidewire (Fig. 3B). At the tip of delivery system of the Niti-S large cell stent slim delivery, the step between the delivery system and guidewire becomes lower than that of the delivery system of the conventional 8-Fr delivery system (Fig. 3C, D). Only two reports have described the efficacy of the Niti-S large cell slim delivery (Taewoong Medical) for hilar multiple SEMS insertion $(35,36)$. Takenaka et al. (36) reported that a second SEMS insertion was achieved without using a dilation catheter. Inoue et al. (35) reported the insertion of three SEMSs, where the combination of the placement of stent-in-stent SEMSs and side-by-side SEMSs was performed. In a report written by Uchida et al. (20), more than three-branched drainage improved the duration of patency in patients with unresectable hilar biliary carcinoma. In that report, six patients received four SEMSs using 7- or 8-Fr devices. However, the detailed Bismuth classification and difficulty encountered with those six cases were not described. In addition, cases with a percutaneous approach were included in the report.

In the present case, a dilation balloon catheter was used when the third stent was inserted. However, the insertion of the third stent was not attempted without a balloon catheter. Therefore, there was a possibility that the third stent might pass through the cells of the first and second stents without a dilation device. In contrast, insertion of the fourth stent did not require a dilation catheter or balloon catheter and was successfully completed within about 20 minutes. Therefore, the Niti-S large cell slim delivery (Taewoong Medical) might be useful for the stent-in-stent insertion of more than three SEMSs.

In conclusion, some patients need placement of multiple biliary SEMSs; however, the drainage of multiple hepatic ducts can be difficult. In this report, a patient required the insertion of four SEMSs to treat a liver abscess. A new SEMS with a wide mesh and thin delivery system was effective for stent-in-stent hilar multiple SEMS insertion.

The authors state that they have no Conflict of Interest (COI). 


\section{Acknowledgements}

We thank all of the staff at the Department of Gastroenterology of Fukushima Medical University, the Department of Endoscopy of Fukushima Medical University Hospital, and the gastroenterology ward of Fukushima Medical University Hospital.

\section{References}

1. Kennedy TJ, Yopp A, Qin Y, et al. Role of preoperative biliary drainage of liver remnant prior to extended liver resection for hilar cholangiocarcinoma. HPB 11: 445-451, 2009.

2. Nuzzo G, Giuliante F, Ardito F, et al. Improvement in perioperative and long-term outcome after surgical treatment of hilar cholangiocarcinoma: results of an Italian multicenter analysis of 440 patients. Arch Surg 147: 26-34, 2012.

3. Wiggers JK, Groot Koerkamp B, Cieslak KP, et al. Postoperative mortality after liver resection for perihilar cholangiocarcinoma: development of a risk score and importance of biliary drainage of the future liver remnant. J Am Coll Surg 223: 321-331 e321, 2016.

4. Takahashi Y, Nagino M, Nishio H, Ebata T, Igami T, Nimura Y. Percutaneous transhepatic biliary drainage catheter tract recurrence in cholangiocarcinoma. Br J Surg 97: 1860-1866, 2010.

5. Kawakami H, Kuwatani M, Onodera M, et al. Endoscopic nasobiliary drainage is the most suitable preoperative biliary drainage method in the management of patients with hilar cholangiocarcinoma. J Gastroenterol 46: 242-248, 2011.

6. Davids PH, Groen AK, Rauws EA, Tytgat GN, Huibregtse K. Randomised trial of self-expanding metal stents versus polyethylene stents for distal malignant biliary obstruction. Lancet 340: 1488-1492, 1992.

7. Lammer J, Hausegger KA, Fluckiger F, et al. Common bile duct obstruction due to malignancy: treatment with plastic versus metal stents. Radiology 201: 167-172, 1996.

8. Mukai T, Yasuda I, Nakashima M, et al. Metallic stents are more efficacious than plastic stents in unresectable malignant hilar biliary strictures: a randomized controlled trial. J Hepatobiliary Pancreat Sci 20: 214-222, 2013.

9. Sangchan A, Kongkasame W, Pugkhem A, Jenwitheesuk K, Mairiang P. Efficacy of metal and plastic stents in unresectable complex hilar cholangiocarcinoma: A randomized controlled trial. Gastrointest Endosc 76: 93-99, 2012.

10. Xia MX, Pan YL, Cai XB, et al. Comparison of endoscopic bilateral metal stent drainage with plastic stents in the palliation of unresectable hilar biliary malignant strictures: Large multicenter study. Dig Endosc. Forthcoming.

11. Chang WH, Kortan P, Haber GB. Outcome in patients with bifurcation tumors who undergo unilateral versus bilateral hepatic duct drainage. Gastrointest Endosc 47: 354-362, 1998.

12. De Palma GD, Galloro G, Siciliano S, Iovino P, Catanzano C. Unilateral versus bilateral endoscopic hepatic duct drainage in patients with malignant hilar biliary obstruction: results of a prospective, randomized, and controlled study. Gastrointest Endosc 53: 547-553, 2001.

13. De Palma GD, Pezzullo A, Rega M, et al. Unilateral placement of metallic stents for malignant hilar obstruction: a prospective study. Gastrointest Endosc 58: 50-53, 2003.

14. Naitoh I, Ohara H, Nakazawa $T$, et al. Unilateral versus bilateral endoscopic metal stenting for malignant hilar biliary obstruction. J Gastroenterol Hepatol 24: 552-557, 2009.

15. Iwano $\mathrm{H}$, Ryozawa $\mathrm{S}$, Ishigaki $\mathrm{N}$, et al. Unilateral versus bilateral drainage using self-expandable metallic stent for unresectable hilar biliary obstruction. Dig Endosc 23: 43-48, 2011.

16. Yasuda I, Mukai T, Moriwaki H. Unilateral versus bilateral endoscopic biliary stenting for malignant hilar biliary strictures. Dig Endosc 25 (Suppl 2): 81-85, 2013.
17. Fukasawa M, Takano S, Shindo H, Takahashi E, Sato T, Enomoto $\mathrm{N}$. Endoscopic biliary stenting for unresectable malignant hilar obstruction. Clin J Gastroenterol 10: 485-490, 2017.

18. Lee $\mathrm{TH}$, Kim $\mathrm{TH}$, Moon $\mathrm{JH}$, et al. Bilateral versus unilateral placement of metal stents for inoperable high-grade malignant hilar biliary strictures: a multicenter, prospective, randomized study (with video). Gastrointest Endosc 86: 817-827, 2017.

19. Teng F, Xian YT, Lin J, Li Y, Wu AL. Comparison of unilateral with bilateral metal stenting for malignant hilar biliary obstruction. Surg Laparosc Endosc Percutan Tech 29: 43-48, 2019.

20. Uchida D, Kato H, Muro S, et al. Efficacy of endoscopic over 3branched partial stent-in-stent drainage using self-expandable metallic stents in patients with unresectable hilar biliary carcinoma. J Clin Gastroenterol 49: 529-536, 2015.

21. Brierley JD, Gospodarowicz MK, Wittekind C. TNM-classification of malignant tumours. Eighth Edition. Wiley-Blackwell, New Jersey, 2017.

22. Kogure $H$, Isayama $H$, Kawakubo $K$, et al. Endoscopic bilateral metallic stenting for malignant hilar obstruction using newly designed stents. J Hepatobiliary Pancreatic Sciences 18: 653-657, 2011.

23. Kogure H, Isayama $H$, Nakai $Y$, et al. Newly designed large cell Niti-S stent for malignant hilar biliary obstruction: a pilot study. Surg Endosc 25: 463-467, 2011.

24. Kogure H, Isayama H, Nakai Y, et al. High single-session success rate of endoscopic bilateral stent-in-stent placement with modified large cell Niti-S stents for malignant hilar biliary obstruction. Dig Endosc 26: 93-99, 2014.

25. Hong W, Sun X, Zhu Q. Endoscopic stenting for malignant hilar biliary obstruction: should it be metal or plastic and unilateral or bilateral? Eur J Gastroenterol Hepatol 25: 1105-1112, 2013.

26. Vienne A, Hobeika E, Gouya H, et al. Prediction of drainage effectiveness during endoscopic stenting of malignant hilar strictures: the role of liver volume assessment. Gastrointest Endosc 72: 728-735, 2010.

27. Takahashi E, Fukasawa M, Sato T, et al. Biliary drainage strategy of unresectable malignant hilar strictures by computed tomography volumetry. World J Gastroenterol 21: 4946-4953, 2015.

28. Lam YH, Wong SK, Lee DW, et al. ERCP and pyogenic liver abscess. Gastrointest Endosc 50: 340-344, 1999.

29. Dull JS, Topa L, Balgha V, Pap A. Non-surgical treatment of biliary liver abscesses: efficacy of endoscopic drainage and local antibiotic lavage with nasobiliary catheter. Gastrointest Endosc 51: 5559, 2000.

30. Sharma BC, Agarwal N, Garg S, Kumar R, Sarin SK. Endoscopic management of liver abscesses and cysts that communicate with intrahepatic bile ducts. Endoscopy 38: 249-253, 2006.

31. Sersté T, Bourgeois N, Vanden Eynden F, Coppens E, Devière J, Le Moine O. Endoscopic drainage of pyogenic liver abscesses with suspected biliary origin. Am J Gastroenterol 102: 1209-1215, 2007.

32. Sharma BC, Garg V, Reddy R. Endoscopic management of liver abscess with biliary communication. Dig Dis Sci 57: 524-527, 2012.

33. Kawakubo K, Kawakami H, Toyokawa Y, et al. Risk factors for technical failure of endoscopic double self-expandable metallic stent placement by partial stent-in-stent method. J Hepatobiliary Pancreat Sci 22: 79-85, 2015.

34. Sugimoto M, Takagi T, Suzuki R, et al. Predictive factors for the failure of endoscopic stent-in-stent self-expandable metallic stent placement to treat malignant hilar biliary obstruction. World J Gastroenterol 23: 6273-6280, 2017.

35. Inoue T, Ibusuki M, Kitano R, Kobayashi Y, Ito K, Yoneda M. A novel large cell-sized stent with slim delivery for combined sideby-side and stent-in-stent placement in malignant hilar biliary obstruction. Endoscopy 52: E104-E105, 2020. 
36. Takenaka M, Nakai A, Kudo M. Novel concept of bared type metallic stent for endoscopic bilateral stent-in-stent placement in patients with hilar malignant biliary obstruction (with video). J Hepatobiliary Pancreat Sci 27: 282-283, 2020.
The Internal Medicine is an Open Access journal distributed under the Creative Commons Attribution-NonCommercial-NoDerivatives 4.0 International License. To view the details of this license, please visit (https://creativecommons.org/licenses/ by-nc-nd/4.0/).

(C) 2021 The Japanese Society of Internal Medicine Intern Med 60: 1871-1876, 2021 